\title{
Monitoring of Fabrication Strain and Temperature During Composite Cure Using Fiber Optic Sensor
}

\author{
Hyun-Kyu Kang, Dong-Hoon Kang, Chang-Sun Hong*, and Chun-Gon Kim \\ Department of Aerospace Engineering, \\ Korea Advanced Institute of Science and Technology (KAIST), \\ 373-1 Kusong-dong, Yusong-gu, Taejon, 305-701, Korea
}

\begin{abstract}
Fabrication strains during cure can lead to warpage or spring-back, which presents difficulties in the assembly of composite structures and residual stresses due to differences of thermal expansion coefficients between fiber and matrix in cooling stage can have intense effects on the mechanical properties of the composite product. The quality of the final composite part is directly affected by cure time, temperature, and pressure. The knowledge of cure process is very helpful to obtain high quality composites at reduced producing cost. Therefore, sensors capable of monitoring the cure process are desired and fiber optic sensors are the good candidate for cure monitoring of composite materials.

In this paper, we present the simultaneous monitoring of the fabrication strain and temperature during the composite cure process by using fiber optic sensors. Fiber Bragg grating/extrinsic Fabry-Perot interferometric (FBG/EFPI) hybrid sensors are used to monitor those measurands. The characteristic matrix of the sensor is analytically derived and measurements can be done without sensor calibration for each experiment. A wavelength-swept fiber laser is utilized as a light source. FBG/EFPI sensors are embedded in graphite/epoxy composite laminates at different direction and different location. We perform the real time monitoring of fabrication strains and temperatures at two points of composite laminates during cure process in an autoclave. Through this experiment, we can provide a basis for the efficient smart processing of composites.
\end{abstract}

Keywords: fabrication strain, temperature, composite cure, FBG/EFPI sensor, cure monitoring

\section{INTRODUCTION}

Composite materials are increasingly being used as engineering materials in aircraft, building, containers, and other structures. Composite structures are fabricated by using a variety of techniques, for example, vacuum bag autoclave technique, hot press, resin transfer molding, pultrusion, and filament winding. Among these techniques, the vacuum bag autoclave molding is used extensively for producing of high-quality aerospace structures. In this technique, generally, the curing cycle of graphite/epoxy (GR/EP) composite consists of 3 stages as following. In stage I, the temperature of the laminate is increased to an intermediate temperature $\left(80^{\circ} \mathrm{C}\right)$ and held for a half hour to decrease the viscosity of the resin. In stage II, the temperature is increased to the final cure temperature $\left(130^{\circ} \mathrm{C}\right)$ and held for two hours. And in the final stage (stage III), the composite is cooled to room temperature. During a chain of curing stages, the composite laminate experiences the heavy temperature change and the fabrication strain develops.

Fabrication strains during cure can lead to significant problems such as warpage or spring-back, which presents difficulties in the assembly of composite structures. Residual stresses due to differences in fiber and matrix expansion coefficients in cooling stage can have intense effects on the mechanical properties of the composite product such as compressive and buckling strength, fracture toughness, and fatigue strength, etc. The quality of the final composite part is directly affected by cure conditions such as cure time, temperature, and pressure. Informations such as temperature, the formation of residual stress, viscosity, degree of cure, and resin flow inside the material itself need to be quantified as a function of time and position within the laminate during cure ${ }^{1}$. The knowledge of cure process is very helpful to obtain high quality composites at reduced producing cost, so it is very important to determine optimum cure condition. Until now, the cure conditions are empirically determined by some trial-and-error tests. But such processes are time-consuming and inefficient. Therefore, there is a demand for sensors that are capable of monitoring of the cure process in situ and at points remote from the surface.

* Correspondence: Email: cshong@kaist.ac.kr; Telephone: +82-42-869-3712; Fax: +82-42-869-3710;

Homepage: http://smartech.kaist.ac.kr

Nondestructive Evaluation of Materials and Composites V, George Y. Baaklini, Eric S. Boltz, 
A prerequisite of any in situ sensor for composite materials is that it must not be detrimental to the operational requirements of the structure. Fiber optic sensors that are easily embedded in composites are the good candidate for cure monitoring of composite materials. Furthermore, the dimensions and orientation of the optical fibers within the prepregs can be selected to have a minimal impact on the mechanical properties of the composite. Also, an advantage of fiber optic sensor technology compared with other cure monitoring techniques such as electrical resistance and dielectric analysis is its low susceptibility to electromagnetic interference. Thus, they can be used to monitor several parameters such as the fabrication strain, and temperature during cure and to evaluate the structural integrity in service after cure.

In literatures for composite cure monitoring using fiber optic sensors, most researches have conducted only the measurement of the fabrication strain using various types of sensors such as extrinsic Fabry-Perot interferometric (EFPI) sensor ${ }^{2-4}$ and fiber Bragg grating (FBG) sensor 5 . Several techniques, however, have been reported for the simultaneous measurement of strain and temperature using FOSs in recent years ${ }^{6-10}$. By using these techniques, the simultaneous monitoring of the fabrication strain and the cure temperature is possible with single fiber optic sensor.

In this paper, we present the simultaneous monitoring of the fabrication strain and temperature during the $\mathrm{Gr} / \mathrm{EP}$ composite cure process by using fiber optic sensors. Fiber Bragg grating/extrinsic Fabry-Perot interferometric (FBG/EFPI) hybrid sensors are used to monitor those measurands. The characteristic matrix of the sensor is analytically derived and measurements can be done without sensor calibration for each experiment. A wavelength-swept fiber laser is utilized as a light source. Two FBG/EFPI sensors are embedded in both graphite/epoxy unidirectional and symmetric cross-ply laminates at different direction and different location. We monitor simultaneously fabrication strains and temperatures at two points inside of composite laminates during cure process in an autoclave.

\section{ANALYTIC RELATIONSHIP FOR FBG/EFPI HYBRID SENSOR}

For the simultaneous measurement of strain and temperature, we need two sensing elements that can supply two equations with respect to two parameters. Many of the approaches have dealt with using grating type sensor, combination of interferometric and polarimetric techniques, fiber Bragg grating/Fabry-Perot interferometric hybrid sensors, and other hybrid type sensors. In most approaches, a number of experiments had to be performed to obtain the characteristic matrices of each sensor. These are time-consuming and may lead to the lack of accurate measurements.

In general, the relationship of the sensor outputs $\phi_{1}$ and $\phi_{2}$ to any combination of strain $\varepsilon$ and temperature change $\Delta T$ can be given by

$$
\left\{\begin{array}{l}
\phi_{1} \\
\phi_{2}
\end{array}\right\}=\left[\begin{array}{ll}
K_{1 \varepsilon} & K_{1 T} \\
K_{2 \varepsilon} & K_{2 T}
\end{array}\right]\left\{\begin{array}{c}
\varepsilon \\
\Delta T
\end{array}\right\}=\mathbf{K}\left\{\begin{array}{c}
\varepsilon \\
\Delta T
\end{array}\right\}
$$

where the components of the matrix $\boldsymbol{K}$ are the strain and temperature sensitivities of the sensor. In order to obtain these components of the characteristics matrix of the sensor, in almost researches a number of experiments have been performed before the application of sensors. This equation is then inverted to yield the strain and the temperature from $\phi_{1}$ and $\phi_{2}$ as following

$$
\left\{\begin{array}{c}
\varepsilon \\
\Delta T
\end{array}\right\}=\left[\begin{array}{ll}
P_{1 \varepsilon} & P_{2 \varepsilon} \\
P_{1 T} & P_{2 T}
\end{array}\right]\left\{\begin{array}{l}
\phi_{1} \\
\phi_{2}
\end{array}\right\}=\mathbf{P}\left\{\begin{array}{l}
\phi_{1} \\
\phi_{2}
\end{array}\right\}
$$

where the matrix $\boldsymbol{P}$ is the inverse matrix of $\boldsymbol{K}$ in (1). However, if specifications of sensors such as gage length vary, experiments must be performed again to obtain components of matrix $\boldsymbol{K}$ for each sensor that has different specifications. The great limitation of method requiring matrix inversion is that the inversion is often poorly conditioned because of a small determinant and consequently gives large errors and low accuracy sometimes. While in many cases it is acceptable to calibrate the sensor, it is more desirable to be able to use the sensor without calibration experiments. In our previous research, we analytically derived the characteristic matrix of FBG/EFPI sensor as following ${ }^{10}$.

Fig. 1 is a schematic diagram of the FBG/EFPI sensor. FBG is encapsulated in a silica capillary tube to be isolated from the external strain. The EFPI cavity was formed between two cleaved fiber ends inserted into the capillary tube. The FBG is in strain-free condition and is only affected by the temperature change within the capillary tube, while EFPI is affected by both thermal and mechanical strains. When a broadband light illuminates with this sensing element, reflected spectrum of the Bragg grating element has a narrow bandwidth with high reflectivity whereas the sinusoidal wave with a fraction of the total optical power is reflected through the EFPI sensor, a low finesse interferometer. 


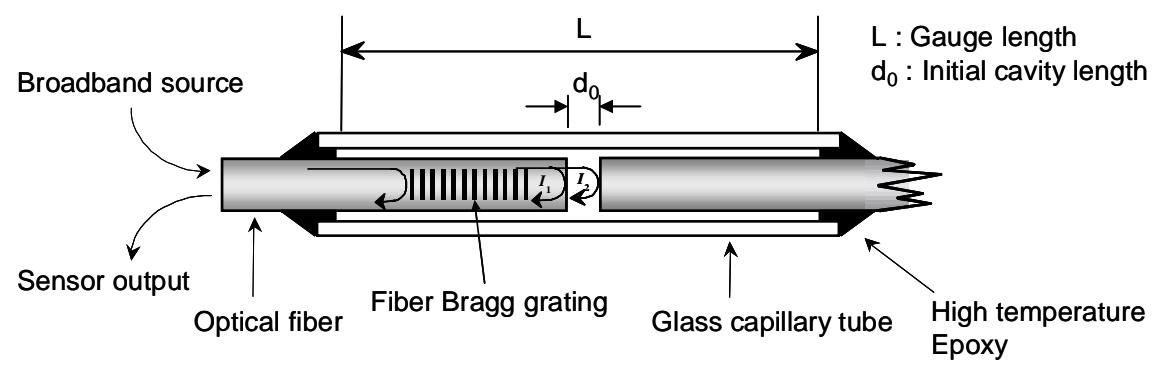

Fig. 1 Configuration of FBG/EFPI sensor.

For a FBG, Bragg condition is given by $\lambda_{B}=2 n_{e} \Lambda$, where $\lambda_{B}$ is the Bragg wavelength of FBG, $n_{e}$ is the effective index of the fiber core, and $\Lambda$ is the grating period. The shift of Bragg wavelength due to the strain and temperature can be expressed as

$$
\left.\Delta \lambda_{B}=\lambda_{B} \mid\left(\alpha_{f}+\xi_{f}\right) \Delta T+\left(1-p_{e}\right) \varepsilon\right]
$$

where $\alpha_{f}$ is the coefficient of thermal expansion (CTE), $\xi_{f}$ is the thermo-optic coefficient, and $p_{e}$ is the strain-optic tensor of optical fiber. For the FBG/EFPI sensor, because the FBG in a glass capillary tube is in strain-free condition, $\varepsilon=0$, (3) can be simplified in terms of only the temperature change as

$$
\Delta \lambda_{B}=\lambda_{B}\left(\alpha_{f}+\xi_{f}\right) \Delta T
$$

Therefore, we can measure the temperature change from the wavelength shift as

$$
\Delta T=\frac{1}{\alpha_{f}+\xi_{f}} \cdot \frac{\Delta \lambda_{B}}{\lambda_{B}}
$$

The response of absolute EFPI(AEFPI) sensor comes from thermal strain as well as applied strain of a structure. When perfectly bonded to structure, the thermal expansion of the sensor is constrained to take on the CTE of the host structure. Therefore, the thermal expansion of FOS bonded to or embedded in a material becomes that of the structure itself. The cavity length, $d_{i}$, at any measuring instant can be measured from peaks of reflected spectrum as

$$
d_{i}=\frac{m \lambda_{1} \lambda_{2}}{2\left(\lambda_{2}-\lambda_{1}\right)} \quad i=0,1,2 \ldots
$$

where $\lambda_{1}$ and $\lambda_{2}$ are two wavelengths that are $2 m \pi$ out of phase and $m$ is an integer. The $i$ is the number of measuring instant, and in case of initial state, $i=0$. If the EFPI sensor has a gage length of $L$, the applied strain can be expressed as

$$
\varepsilon=\frac{d_{n}-d_{0}}{L}=\frac{\Delta d}{L} .
$$

where $\Delta d$ is the change of cavity length, and $d_{n}, d_{0}$ are the final and initial cavity lengths, respectively. When the temperature change and strain are applied to structures with embedded or attached an FBG/EFPI sensor, the lengths of internal optical fibers in capillary tube are changed with their CTE only by temperature change while external capillary tube subjects to both thermal and mechanical strains of structures. Therefore, we can measure the total strain applied to structure through the variation of a reflected spectrum by the difference of length change between sum of two internal fibers in tube and the capillary tube. The strain measured from the EFPI interferometric signal can be described by

$$
\varepsilon_{\text {mea }}=\varepsilon_{\text {tot }}-\varepsilon_{f, T}=\frac{\Delta d}{L}
$$


where $\varepsilon_{\text {tot }}$ is the sum of mechanical and thermal strain of structure, $\varepsilon_{f, T}$ is the thermal strain of internal optical fibers, respectively. We want to obain the total strain of structure, that is, the strain of the capillary tube. From (8) we can get the equation about total strain as

$$
\varepsilon_{s t}=\frac{\Delta d}{L}+\varepsilon_{f, T}=\frac{\Delta d}{L}+\frac{L-d_{0}}{L} \cdot \alpha_{f} \cdot \Delta T
$$

Substituting (5) into (9) leads to

$$
\varepsilon_{s t}=\frac{\Delta d}{L}+\frac{L-d_{0}}{L} \cdot \frac{\alpha_{f}}{\alpha_{f}+\xi_{f}} \cdot \frac{\Delta \lambda_{B}}{\lambda_{B}}
$$

By combining (5) and (10), we can yield the relationships of the sensor outputs to measurands as

$$
\left\{\begin{array}{c}
\varepsilon \\
\Delta T
\end{array}\right\}=\left[\begin{array}{cc}
\frac{1}{L} & \frac{L-d_{0}}{L} \cdot \frac{\alpha_{f}}{\alpha_{f}+\xi_{f}} \cdot \frac{1}{\lambda_{B}} \\
0 & \frac{1}{\alpha_{f}+\xi_{f}} \cdot \frac{1}{\lambda_{B}}
\end{array}\right]\left\{\begin{array}{c}
\Delta d \\
\Delta \lambda_{B}
\end{array}\right\}
$$

In (2), components of characteristic matrix are $P_{1 \varepsilon}=\frac{1}{L}, P_{2 \varepsilon}=\frac{L-d_{0}}{L} \cdot \frac{\alpha_{f}}{\alpha_{f}+\xi_{f}} \cdot \frac{1}{\lambda_{B}}, P_{1 T}=0$, and $P_{2 T}=\frac{1}{\alpha_{f}+\xi_{f}} \cdot \frac{1}{\lambda_{B}}$ and sensor outputs are $\phi_{1}=\Delta d$ and $\phi_{2}=\Delta \lambda_{B}$, respectively.

From (11), it should be noted that the characteristic matrix of a sensor can be determinted by sensor specifications such as gage length, initial cavity length, Bragg wavelength of FBG, the CTE and thermal-optic coefficient of optical fibers. The strain and the temperature can be easily determined by measuring the change of cavity length for the strain and the wavelength shift for the temperature. In the conventional method, all sensors with different specifications must be calibrated to obtain the characteristic matrix before the field use. However, we can generate simply the matrix with known specifications of sensors using (11) and measure directly and simultaneously the strain and the temperature of structures.

\section{EXPERIMENTS}

\subsection{Lay-up of composite laminates with embedded FBG/EFPI sensors}

A $10 \mathrm{~mm}$ long FBG was encapsulated in a silica capillary tube $(140 / 300 \mu \mathrm{m})$ to be isolated from the external strain. The EFPI cavity was formed between the fiber end face near to the FBG and gold-coated fiber end surface inserted into the opposite end of the capillary tube. Specifications of sensor embedded in composite laminates are shown in Table I. Substituting specifications of the sensor in TABLE I into (11), we can obtain analytically the components of characteristic matrices of sensors as shown in Table II.

Table I. Specifications of FBG/EFPI sensors.

\begin{tabular}{c||c||c||c|c||c|c}
\hline \hline \multirow{2}{*}{ Sensor Spec. } & \multirow{2}{*}{ Symbol } & \multirow{2}{*}{ Unit } & \multicolumn{2}{|c||}{$\left[0_{12} /\{0\} / 0_{12}\right]_{\mathrm{T}}$} & \multicolumn{2}{c}{$\left[0_{6} / 90_{6} /\{90\} / 90_{6} / 0_{3} /\{0\} / 0_{3}\right]_{\mathrm{T}}$} \\
\cline { 4 - 7 } & & & Sensor 1 & Sensor 2 & Sensor 3 & Sensor 4 \\
\hline \hline Gage length & $L$ & $m m$ & 24.3 & 24.5 & 24.8 & 23.9 \\
\hline Bragg wavelength & $\lambda_{B}$ & $n m$ & 1532.003 & 1532.441 & 1532.050 & 1532.070 \\
\hline Initial cavity length & $d_{0}$ & $\mu m$ & 664.56 & 642.66 & 660.55 & 680.55 \\
\hline CTE of optical fiber & $\alpha_{f}$ & $p p m /{ }^{\circ} \mathrm{C}$ & \multicolumn{2}{|c|}{0.5} & \multicolumn{2}{c}{0.5} \\
\hline Thermo-optic coefficient & $\xi_{f}$ & $\mathrm{ppm} /{ }^{\circ} \mathrm{C}$ & \multicolumn{2}{|c|}{6.2} & \multicolumn{2}{c}{6.2} \\
\hline \hline
\end{tabular}


Table II. Components of characteristic matrices of sensors.

\begin{tabular}{c||c||c|c||c|c}
\hline \hline \multirow{2}{*}{$\begin{array}{c}\text { Components of } \\
\text { characteristic matrices }\end{array}$} & \multirow{2}{*}{ Unit } & \multicolumn{2}{c||}{$\left[0_{12} /\{0\} / 0_{12}\right]_{\mathrm{T}}$} & \multicolumn{2}{|c}{$\left[0_{6} / 90_{6} /\{90\} / 90_{6} / 0_{3} /\{0\} / 0_{3}\right]_{\mathrm{T}}$} \\
\cline { 2 - 6 } & & Sensor 1 & Sensor 2 & Sensor 3 & Sensor 4 \\
\hline \hline$P_{I \varepsilon}$ & $\mu \varepsilon / \mu \mathrm{m}$ & 41.15 & 40.82 & 40.32 & 41.84 \\
\hline$P_{2 \varepsilon}$ & $\mu \varepsilon / n m$ & 47.38 & 47.42 & 47.41 & 47.32 \\
\hline$P_{I T}$ & ${ }^{\circ} \mathrm{C} / \mu \mathrm{m}$ & 0 & 0 & 0 & 0 \\
\hline$P_{2 T}$ & ${ }^{\circ} \mathrm{C} / \mathrm{nm}$ & 97.42 & 97.40 & 97.42 & 97.42 \\
\hline \hline
\end{tabular}

Fig. 2 shows schematic diagrams of the $\left[0_{12} /\{0\} / 0_{12}\right]_{\mathrm{T}}$ unidirectional and $\left[0_{6} / 90_{6} /\{90\} / 90_{6} / 0_{3} /\{0\} / 0_{3}\right]_{\mathrm{T}}$ cross-ply laminates indicating the direction and location of fiber optic sensors and the thermocouple. Laminates were made of CU-125 NS graphite/epoxy prepreg (HFG Co.). Sensor 1 and sensor 2 were embedded in the center of the $\left[0_{12} /\{0\} / 0_{12}\right]_{\mathrm{T}}$ laminate between the $12^{\text {th }}$ and $13^{\text {th }}$ plies. Sensor 1 was embedded parallel and sensor 2 was embedded perpendicular to reinforcing fibers, respectively. Sensor 3 was embedded between the $21^{\text {th }}$ and $22^{\text {th }}$ plies and Sensor 4 was embedded between the $12^{\text {th }}$ and $13^{\text {th }}$ plies of the $\left[0_{6} / 90_{6} /\{90\} / 90_{6} / 0_{3} /\{0\} / 0_{3}\right]_{\mathrm{T}}$ laminate so that both sensors could be embedded parallel to reinforcing fibers. Ktype thermocouple was used to serve as a reference source for the temperature.

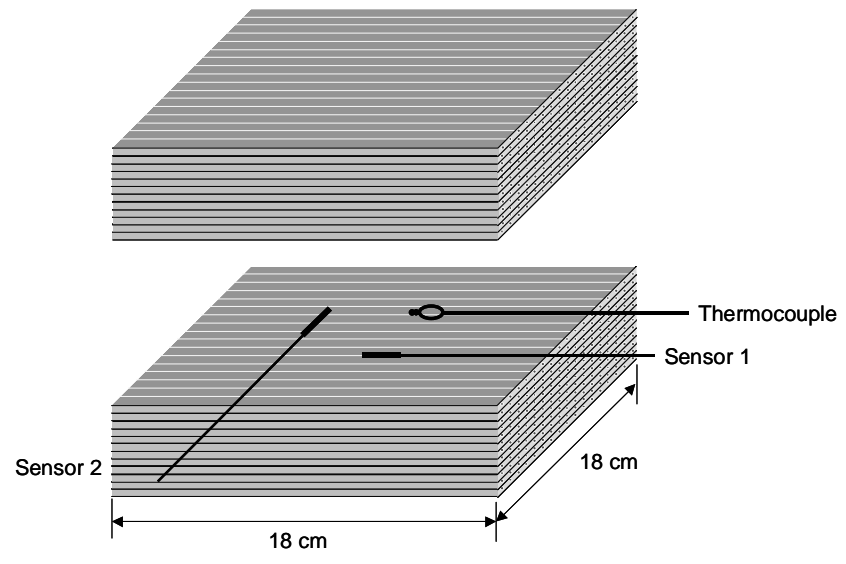

(a) $\left[0_{12} /\{0\} / 0_{12}\right]_{\mathrm{T}}$.

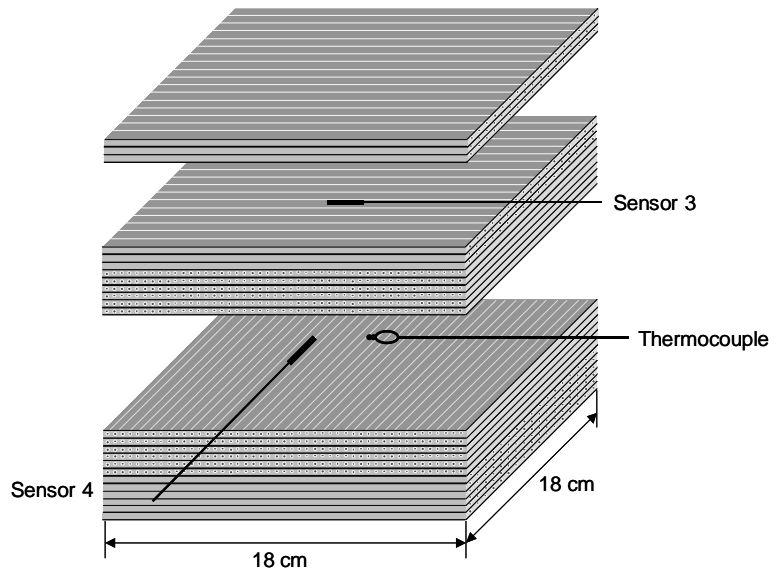

(b) $\left[0_{6} / 90_{6} /\{90\} / 90_{6} / 0_{3} /\{0\} / 0_{3}\right]_{\mathrm{T}}$.

Fig. 2 Directions and locations of the optical fiber sensors embedded in laminates.

\subsection{Experimental Setup}

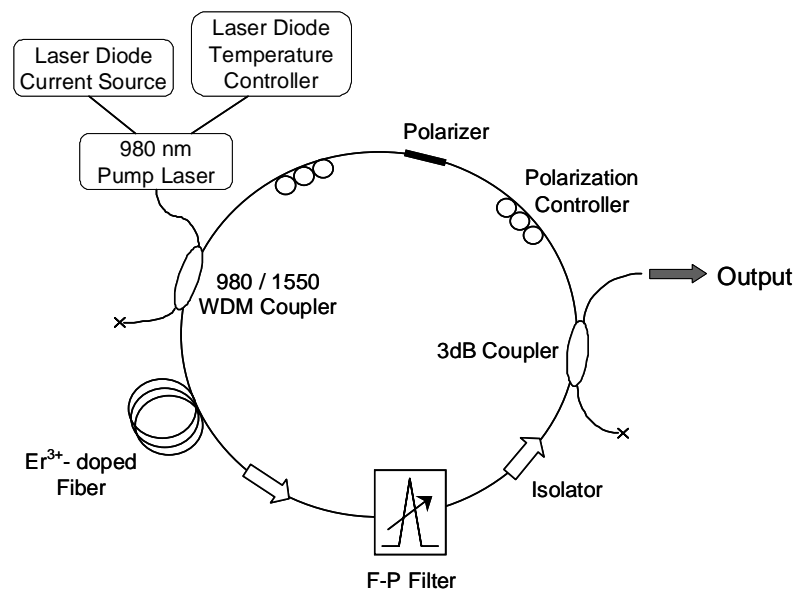

Fig. 3 Schematic diagram of wavelength-swept fiber laser.

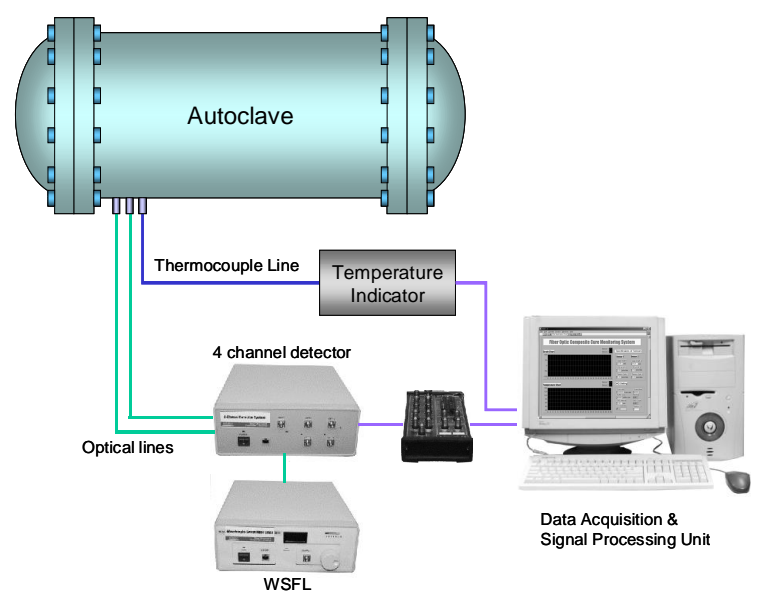

Fig. 4 Experimental Setup. 
A wavelength-swept fiber laser (WSFL) ${ }^{11}$ was used to interrogate the sensor as Fig. 3. The WSFL was in a unidirectional ring configuration with isolators, a 3- $d B$ output coupler, and an $\mathrm{Er}^{3+}$-doped fiber pumped by a laser diode at $980 \mathrm{~nm}$. An FabryPerot (F-P) tunable filter was used as the intracavity scanning filter and had a 3-dB bandwidth of $0.27 \mathrm{~nm}$ and a free spectral range of $58 \mathrm{~nm}$. We modulate the F-P filter with a triangular waveform to produce a wavelength sweep over $35 \mathrm{~nm}$ from 1525 to $1560 \mathrm{~nm}$ at $170 \mathrm{~Hz}$ repetition rate. The output power of the WSFL was over 1000 times as large as that of amplified spontaneous emission (ASE) of an LD-pumped $\mathrm{Er}^{3+}$-doped fiber (EDF) ${ }^{12}$.

The experimental arrangement is depicted in Fig. 4. The composite laminate with embedded FBG/EFPI sensors and thermocouple was placed in autoclave and was fabricated by the curing cycle in Fig. 5. Two optical fibers and thermocouple line were extracted through holes in the wall of the autoclave and connected to the data acquisition and processing system. The reflected signals of fiber optic sensors were acquired through DAQ board, and processed and saved by a signal processing program written in LabVIEW ${ }^{\circledR}$ software.

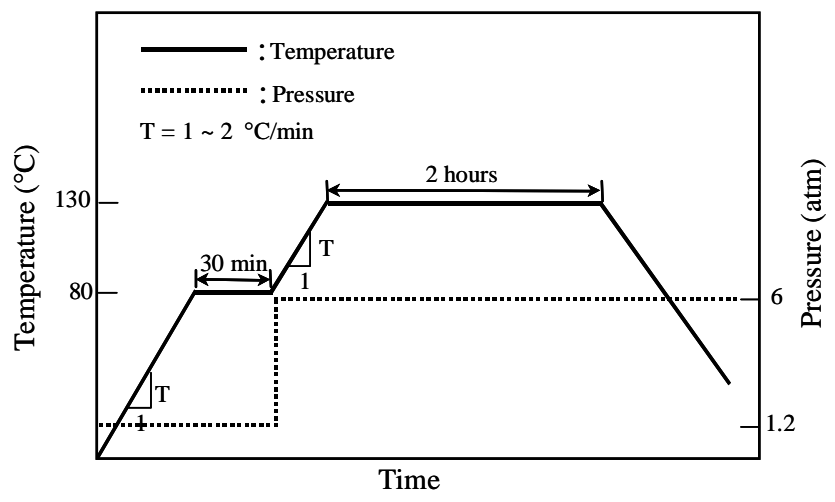

Fig. 5 Typical curing cycle of graphite/epoxy composite In the autoclave moding.

\subsection{Results and discussions}

The plots of strains and temperatures measured by the FOSs during the cure of the $\left[0_{12} /\{0\} / 0_{12}\right]_{\mathrm{T}}$ laminate are given in Fig. 6 . Fig. 6 presents (a) the strain in the $0^{\circ}$ direction measured by sensor 1, (b) the strain in the $90^{\circ}$ direction measured bysensor 2 , and (c) temperatures measured by both sensors. The compressive strains developed abruptly in the transverse direction during the cooling stage. The final compressive strains in the longitudinal and transverse direction were approximately $-140 \mu \varepsilon$ and $-4000 \mu \varepsilon$, respectively. From Fig. 6, it is shown that the strain in the transverse direction is significantly larger than the strain in the longitudinal direction. This is because the properties in the transverse direction are dominated by the epoxy matrix which should exhibit large thermal and chemical deformations during cure. As shown in Fig. 6(c), The temperatures measured by fiber optic sensors have good agreement with the temperature measured by thermocouple.

The plots of strains and temperatures measured by the fiber optic sensors during the cure of the $\left[0_{6} / 90_{6} /\{90\} / 90_{6} / 0_{3} /\{0\} / 0_{3}\right]_{\mathrm{T}}$ laminate are given in Fig. 7. Fig. 7 illustrates (a) strains measured by sensor 3 and 4, and (b) temperatures measured by both sensors. Compressive strains were generated from stage II and large compressive strains grew during the cooling stage. The magnitudes of final compressive strains are approximately $-400 \mu \varepsilon$ in the $0^{\circ}$ direction and $-450 \mu \varepsilon$ in the $90^{\circ}$ direction. As shown in Fig. 7(b), good correlations were found between temperatures measured by fiber optic sensors and thermocouple. Since the unidirectional composite plies have the anisotropic property, they will shrink more in the transverse direction than in the reinforcing fiber direction due to thermal and chemical shrinkage. A fabricated composite laminate acts as a single material, because the bending thickness between plies is very thin and the bonding is as sumed as a perfect bonding. Therfore, the individual plies are not free to deform and are restrained from deformation by adjacent plies $^{2}$. We can see that the strains measured by both sensors in the symmetric cross-ply laminate in Fig. 7 were similar each other and were larger than the strain in the reinforcing fiber direction and less than the strain in the transverse direction of the unidirectional laminate in Fig. 6.

From experimental results, it is found that fiber optic sensors are capable of monitoring of the cure process in situ and at points remote from the surface. This method will be able to supply the informations about cure process and be used to find the optimal cure condition. 


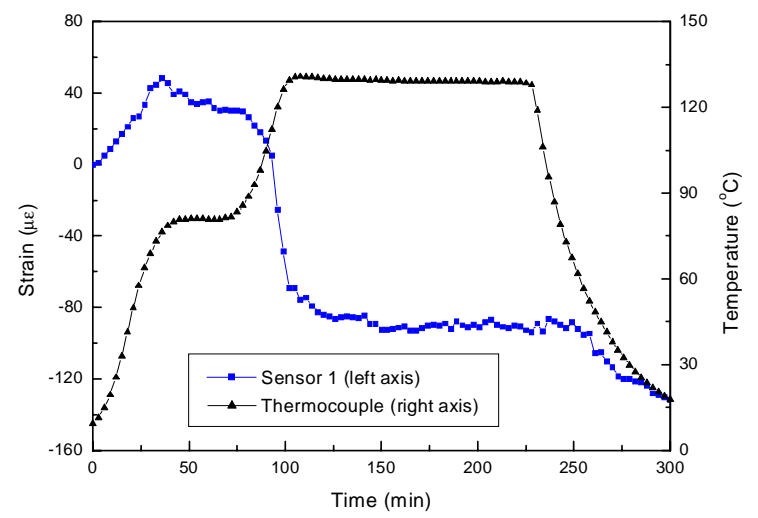

(a) Strain measured by sensor 1 .

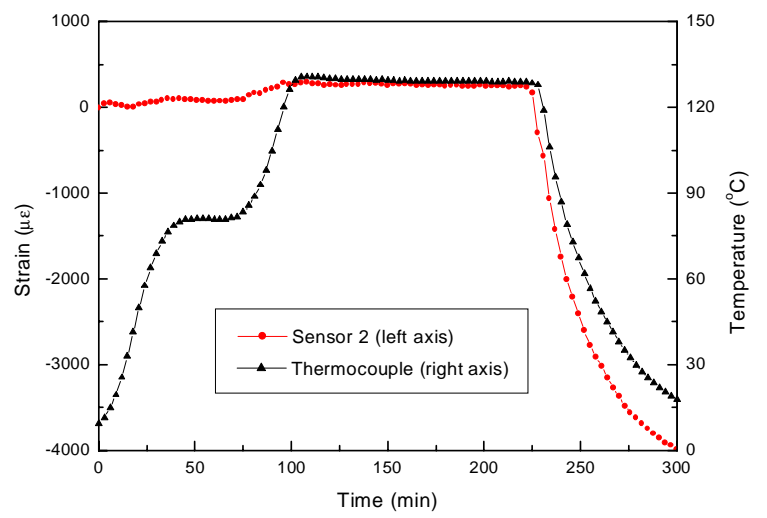

(a) Strain measured by sensor 2 .

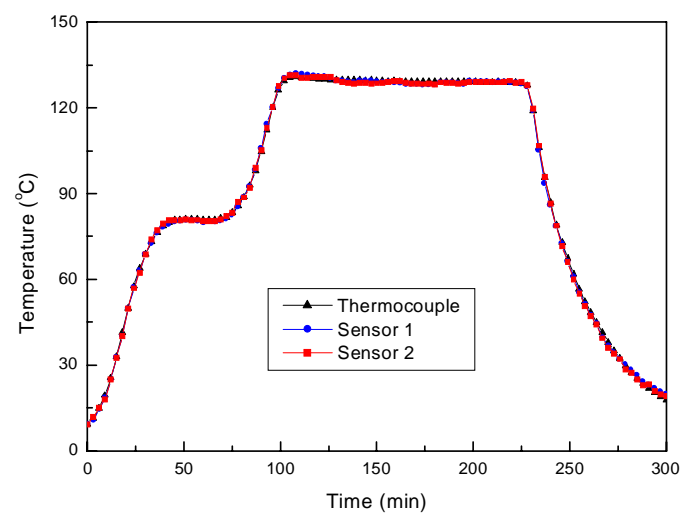

(c) Temperature measurements.

Fig. 6 Strain and temperature measurements in the $\left[0_{12} /\{0\} / 0_{12}\right]_{T}$ laminate.

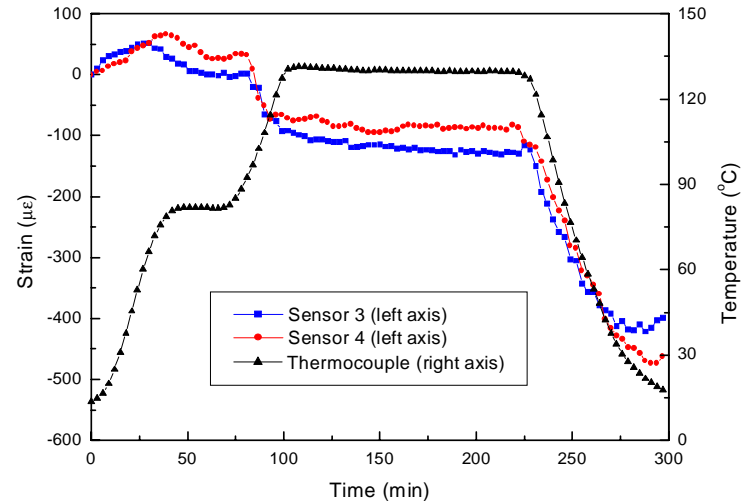

(a) Strain measurements.

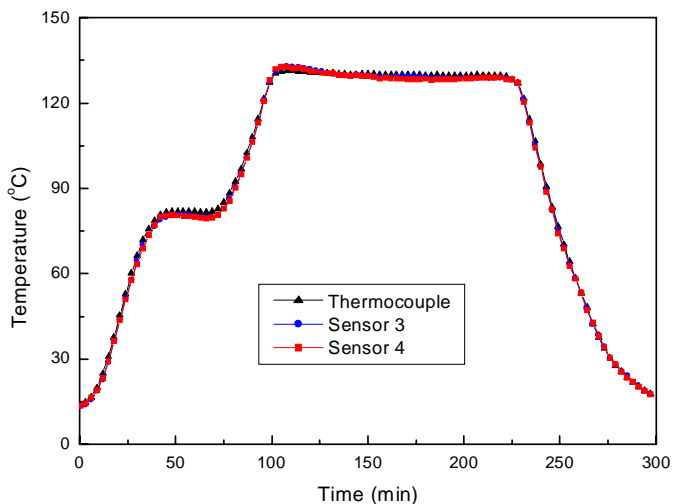

(b) Temperature measurements.

Fig. 7 Strain and temperature measurements in the $\left[0_{6} / 90_{6} /\{90\} / 90_{6} / 0_{3} /\{0\} / 0_{3}\right]_{\mathrm{T}}$ laminate. 


\section{CONCLUSIONS}

In order to monitor the fabricaion strain and the temperature during the the graphite/epoxy composite cure, we used the FBG/EFPI hybrid sensors which can measure the strain and temperature simultaneously. The characteristic matrices of sensors were analytically obtained using their specifications. Fiber optic sensors and thermocouple were embedded in unidirectional and symmetric cross-ply composite laminates, which were fabricated in the autoclave. Embedded FBG/EFPI sensors could simultaneously monitor the fabrication strain and temperature and it is found that these sensors have the potential for monitoring the internal residual strain and terperature during the composite cure. Through this experiment, we can provide a basis for the efficient smart processing of composites.

\section{ACKNOWLEDGEMENT}

The authors would like to thank the Ministry of Science and Technology, Korea, for the financial support by a grant from the Critical Technology 21 project.

\section{REFERENCES}

1. S. S. J. Roberts and R. Davidson, "Cure and Fabrication Monitoring of Composite Materials with Fibre-Optic Sensors," Composite Science and Technology, Vol. 49, pp.265-276, 1993.

2. C. M. Lawrence, D. V. Nelson, T. E. Bennett, and J. R. Spingarn, "An Embedded Fiber Optic Sensor Method for Determining Residual Stresses in Fiber-Reinforced Composite Materials," Journal of Intelligent Material Systems and Structures, Vol. 9, No. 10, pp.788-799, 1998.

3. J. Y. Chen, S. V. Hoa, C. K. Jen, and H. Wang, "Fiber-Optic and Ultrasonic Measurements for In-situ Cure Monitoring of Graphite/Epoxy Composite," Journal of Composite Materials, Vol. 33, No. 20, pp.1860-1881, 1999.

4. K. Osaka, T. Kosaka, Y. Asano, and T. Fukuda, "Measurement of Internal Strains in FRP Laminate with EFPI Optical Fiber Sensor During Autoclave Molding; Measurement in Off-axis Directions," Proceedings of the Second AsianAustralasian Conference on Composite Materials (ACCM-2000), C. S. Hong and C. G. Kim, Editor, Kyongju, Korea, pp.1117-1122, August 2000.

5. V. Dewynter-Marty, P. Ferdinand, E. Bocherens, R. Carbone, H. Beranger, S. Bourasseau, M. Dupont, and Balageas, "Embedded Fiber Bragg Grating Sensors for Industrial Composite Cure Monitoring," Journal of Intelligent Material Systems and Structures, Vol. 9, No. 10, pp.785-787, 1998

6. M. G. Xu, J.-L. Archambault, L. Reekie and J. P. Dakin, "Discrimination between strain and temperature effects using dual-wavelength fibre grating sensors", Electronics Letters, Vol.30, No.13, pp.1085-1087, June 1994.

7. H. J. Patrick, G. M. Williams, A. D. Kersey, J. R. Pedrazzani, and A. M. Vengsarkar, "Hybrid fiber Bragg grating / long period fiber grating sensor for strain / temperature discrimination," IEEE Photonics Technology Letters, Vol. 8, No. 9, pp. 1223-1225, September 1996.

8. V. Bhatia, D. Campbell, R. O. Claus, and A. M. Vengsarkar "Simultaneous strain and temperature measurement with long-period gratings", Optics Letters, Vol.22, No. 9, pp.648-650, May 1997.

9. X. D. Jin and J. S. Sirkis, J. K. Chung and V. S. Venkat, "Embedded in-line fiber eltalon/Bragg grating hybrid sensor to measure strain and temperature in a composite beam," J. of Intelligent Material Systems and Structures, Vol.9, No.3, pp.171-181, March 1998.

10. H. K. Kang, C. Y. Ryu, H. J. Bang, C. S. Hong, and C. G. Kim, "Simultaneous Measurement of Strain and Temperature Using FBG/EFPI Hybrid Sensor," Journal of Lightwave Technology, Submitted, 2001.

11. S. H. Yun, D. J. Richardson, and B. Y. Kim, "Interrogation of fiber grating sensor arrays with a wavelength-swept fiber laser," Optics Letters, Vol. 23, No. 11, pp. 843-845, June 1998.

12. C. S. Hong, C. Y. Ryu, B. Y. Koo, C. G. Kim, S. H. Yun, "Strain monitoring of smart bridge using fiber Bragg grating sensor system with wavelength-swept fiber laser," in Smart Structures and Materials 2000: Smart Systems for Bridges, Structures, and Highways, S. C. Liu, Editor, Proc. SPIE Vol. 3988, pp. 371-379, March 2000. 